\title{
Method for Estimation and Correction of Perspective Distortion of Electroluminescence Images of Photovoltaic Panels
}

Mantel, Claire; Villebro, Frederik; Parikh, Harsh Rajesh; Spataru, Sergiu; Benatto, Gisele Alves dos Reis; Sera, Dezso; Poulsen, Peter Behrensdorff; Forchhammer, Søren

Published in:

IEEE Journal of Photovoltaics

Link to article, DOI:

10.1109/JPHOTOV.2020.3019949

Publication date:

2020

Document Version

Peer reviewed version

Link back to DTU Orbit

Citation (APA):

Mantel, C., Villebro, F., Parikh, H. R., Spataru, S., Benatto, G. A. D. R., Sera, D., Poulsen, P. B., \&

Forchhammer, S. (2020). Method for Estimation and Correction of Perspective Distortion of Electroluminescence Images of Photovoltaic Panels. IEEE Journal of Photovoltaics, 10(6), 1797-1802.

https://doi.org/10.1109/JPHOTOV.2020.3019949

\section{General rights}

Copyright and moral rights for the publications made accessible in the public portal are retained by the authors and/or other copyright owners and it is a condition of accessing publications that users recognise and abide by the legal requirements associated with these rights.

- Users may download and print one copy of any publication from the public portal for the purpose of private study or research.

- You may not further distribute the material or use it for any profit-making activity or commercial gain

- You may freely distribute the URL identifying the publication in the public portal 


\title{
Method for Estimation and Correction of Perspective Distortion of Electroluminescence Images of Photovoltaic Panels
}

\author{
Claire Mantel ${ }^{\circledR}$, Associate Member, IEEE, Frederik Villebro, Harsh Rajesh Parikh ${ }^{\circledR}$, \\ Sergiu Spataru ${ }^{\circledR}$, Member, IEEE, Gisele A. dos Reis Benatto ${ }^{\circledR}$, Dezso Sera ${ }^{\circledR}$, Senior Member, IEEE, Peter B. \\ Poulsen ${ }^{\circledR}$, and Søren Forchhammer ${ }^{\circledR}$, Member, IEEE
}

\begin{abstract}
The number of photovoltaic panels installed globally is continuously growing, requiring an automatic inspection procedure for operation and maintenance. Drones can be a useful tool to this aim as they enable fast acquisition of various imaging modalities: visual, infrared, or electroluminescence (EL). Image distortions due to perspective must be corrected to allow further automatic processing. It can be done by estimating the corresponding rotation angles to control the camera gimbal or as postprocessing to rectify the images. This article presents two methods to achieve both goals by identifying known points in the acquired image. The first method detects the four panel corners, whereas the second method finds the corners of each cell. The performance evaluation is performed first quantitatively on a validation dataset composed of $113 \mathrm{EL}$ images and their corresponding ground-truth orientations. A qualitative evaluation shows satisfying performance of the rectification similarly for both methods. The quantitative performance is varying for each rotation axis. The average absolute error is $2.78^{\circ}$ along the $x$-axis, $2.64^{\circ}$ along the $y$-axis, and $1.28^{\circ}$ along the $z$-axis for the panel method and $3.26^{\circ}, 2.05^{\circ}$, and $1.24^{\circ}$ for the cell method. As a proof of concept, a final test on drone-acquired EL images shows good performance for the image rectification in real-life conditions.
\end{abstract}

Index Terms-Angle estimation, corner detection, electroluminescence (EL), homography, imaging, perspective correction, perspective distortion.

\section{INTRODUCTION}

$\mathbf{T}$ HE photovoltaic (PV) capacity installed worldwide presents a near exponential growth, reaching $633 \mathrm{GW}$ in 2019 and estimated to reach $750-780 \mathrm{GW}$ in 2020 [1]. This

Manuscript received May 14, 2020; revised July 27, 2020; accepted August 21,2020 . This work was carried out in the framework of the project "DronEL" funded by Innovation Fund Denmark under Grant 6154-00012B. (Corresponding author: Claire Mantel.)

Claire Mantel, Frederik Villebro, Sergiu Spataru, Gisele A. dos Reis Benatto, Peter B. Poulsen, and Søren Forchhammer are with the Department of Photonics Engineering, Denmark Technical University, 2800 Kgs Lyngby, Denmark (e-mail: clma@fotonik.dtu.dk; frevi@fotonik.dtu.dk; sersp@ fotonik.dtu.dk; garb@fotonik.dtu.dk; ppou@fotonik.dtu.dk; sofo@fotonik.dtu.dk).

Harsh Rajesh Parikh is with the Department of Energy Technology, Aalborg University, 9220 Aalborg, Denmark (e-mail: hrp@et.aau.dk).

Dezso Sera is with the Science and Engineering Faculty, Queensland University of Technology, Brisbane, QLD 4000, Australia (e-mail: dezso.sera@qut.edu.au).

Color versions of one or more of the figures in this article are available online at http://ieeexplore.ieee.org.

Digital Object Identifier 10.1109/JPHOTOV.2020.3019949 growth is to be linked to the drop in multisilicon PV modules price from over $2 \$$ per watt in 2010 to just over $0.2 \$$ in 2019 [2]. This trend in price decrease is also found in the operation and maintenance costs, where the pressure for price reduction makes automation and digitalization necessary [3]. To that aim, drones have been used for visual and infrared imaging of PV plants [4]. The more novel and detailed near-infrared/electroluminescence (EL) PV imaging technique has recently been demonstrated on a drone to achieve daylight inspection of PV panels [5]. Although PV imaging tools can be used to inspect many panels in short time, they are mostly qualitative diagnostic techniques. Detecting, quantifying, and reporting PV panel failures, from tens of thousands of panel images, require automated image analysis to be feasible and cost efficient. Yet, many issues are still to be resolved to achieve a fully automated inspection pipeline both realized online on the drone but also offline in postprocessing.

One example of such processing necessary on both sides is the estimation and correction of the distortion created in the image by perspective. This perspective distortion, resulting in the panel appearing not rectangular, stems from the location and rotation of the camera in regard to the imaged panel. Indeed, in real inspection conditions, the optimal position of the camera in regard to the imaged panel cannot be guaranteed due to shade from the drone on the panel or spatial restrictions, etc. Furthermore, during aerial image acquisition, the drone/camera system is flying in the air with wind and turbulence greatly influencing its position. Correction is then necessary to obtain the true shape of the panel. This enables processing of several images, which is necessary to obtain good quality in outdoor setting [6] but also to retrieve the true shape of defects presents in the panel, necessary to automatically detect them [7]. First, it will be useful as on-drone processing where the estimation of the rotation angles between the camera and the panel can command the camera gimbal to set it orthogonally (or as close as possible) to the imaged panel. After this first step of reducing the perspective distortion is done on-board, the remaining distortion can be corrected offline as postprocessing.

This article presents a method to achieve those two goals: the estimation of angles between the camera and the panel, as well as its correction. It builds on the conference paper [8] that was presenting a method for the correction based on the detection of 
the four corners of a PV panel and develops it by adding:

1) a second method based on detecting the corners of each cell (as opposed to each module in the conference paper);

2) the estimation of rotation angles with regard to the camera to allow gimbal control; and

3) a quantitative validation for both estimation and correction.

The remainder of this article is organized as follows. Section II will focus on formulating the problem and presenting the corresponding state-of-the-art. Then, the two proposed methods will be introduced in Section III and their performance evaluated in Section IV. Finally, Section V concludes this article.

\section{PROBlem Formulation AND PREVIOUS WORK}

The problem of perspective correction is inherent to all applications involving image acquisition "outside of the laboratory," i.e., from a noncontrolled location with regard to the target imaged [9]. Solving it is required to allow any subsequent automatic processing, for applications as diverse as architectural modeling [10] or optical character recognition [11]. In the case of PV panels, one approach is to identify corners in the acquired image and rectify the image, so those corners are mapped to a rectangle or a square depending on the characteristics of the PV panel [12], and an implementation requiring manual marking of the panel corners can be found [13]. An automatic method was designed in [14] for "small angle rotation" and panels containing "moderate degradation of the outer cell." As the authors assume that the panel is the main structure present in the image, their method cannot generalize to outdoor daylight acquisition. A method to automatically detect the four corners of a PV panel and afterward rectify the image correspondingly has been presented in [8]. It takes advantage of the highly structured aspect of PV panels in images to achieve corner detection and then uses the direct linear transformation (described, e.g., in [15]) to rectify the image. This article extends our paper [8] in three directions: improving the robustness by using more data points (i.e., cell corners instead of panel corners), retrieving the corresponding rotation angles, which could be used to orientate the camera better (e.g., by a gimbal on a drone), and validating the methods both quantitatively and qualitatively to obtain a better estimation of the performance of both proposed methods.

Formally, let $\mathbf{P}_{i}=\left(P_{i x}, P_{i y}\right)$, where $i \in 1,2, \ldots, n$, with $n \in$ $\mathbb{N}$ being the coordinates of known points of the considered panel in the image plane, with $\mathbf{P}_{i} \in \mathbb{R}^{2}$. The known points $\mathbf{P}_{i}$ correspond to the points $\mathbf{C}_{i}$ in a defined perspective corrected image, i.e., orthogonal to the panel and acquired so that the top border of the panel is horizontal: the coordinates of the set $\mathbf{C}_{i}$ are, therefore, defined to represent a square or a rectangle, depending on the number of rows and columns of the panel. The set of points $\mathbf{C}_{i}$ contains the same number of points as the set $\mathbf{P}_{i}$ and all are coplanar, so according to the fundamental theorem of projective geometry [15], there exists a transformation $\mathbf{H}$ such that

$$
\left[\begin{array}{c}
\mathbf{C}_{\mathbf{i x}} \\
\mathbf{C}_{i y} \\
1
\end{array}\right]=\mathbf{H}\left[\begin{array}{c}
\mathbf{P}_{\mathbf{i x}} \\
\mathbf{P}_{i y} \\
1
\end{array}\right] \quad \mathbf{H}=\left[\begin{array}{lll}
h_{11} & h_{12} & h_{13} \\
h_{21} & h_{22} & h_{23} \\
h_{31} & h_{32} & h_{33}
\end{array}\right]
$$

Correcting for the distortions due to perspective is, therefore, equivalent to determining the homography transformation $\mathbf{H}$. $\mathbf{H}$ is defined up to a scaling factor, so it is possible to set $h_{33}=$ 1 , and a total of eight degrees of freedom remain, resulting in a constraint of at least four points identified in both planes: $n \geq 4$.

\section{Method}

The aim of our method is to identify at least four known points in the acquired image, for which the location in the rectified image is known. Those points will allow determining the homography $\mathbf{H}$ [see (1)], and then be used to rectify the image as well as to retrieve the angle rotation between the camera and the panel. This section first presents the two methods to detect points: either the four corners of the module or the corners of each of the cells in Section III-A before the homography estimation and retrieval of the corresponding rotation angle in Section III-B.

Two preprocessing steps are first applied on all images. The first step consists of normalizing the considered image within the range [0-1]. The second step aims at computing the magnitude of the gradients present in the image. To do so, Sobel filters are applied on the $x$ - and $y$-axes, and at each pixel, the L2-norm represents the magnitude of the gradient. The resulting image is illustrated in Fig. 1(a).

\section{A. Line Detection and Corner Computation}

The chosen approach is to define corners as the intersections between lines, both for the panel corners and the cell corners. First, lines are located by rotating the preprocessed image at different angles and summing the pixel values along both the $x$ - and $y$-axes in order to locate horizontal as well as vertical lines. Lines parallel to the axis in question after rotation will be visible as peaks in this summation. Each summation is added as a row in a matrix $\mathbf{R}$ for all rotations with $\mathbf{R} \in \mathbb{R}^{r d}$, where $r$ is the number of rotation angles investigated and $d$ is the number of pixels in the axis. This matrix is reduced to a maximization vector $V_{x}$ or $y$ with $d$ elements by finding the maximum of each row. Thus, the maximization vector $V_{x}$ or $y$ contains the maximum value obtained for each pixel index of all the investigated angles. The maximization vectors $V_{x}$ and $V_{y}$ corresponding to the edge image from Fig. 1(a) are illustrated in Fig. 1(c) and (b), respectively. It is then trivial to locate the index and angle at which a line is present from the maximization vector $V_{x}$ or $y$ along with the rotation matrix $\mathbf{R}$.

In practice, images are first rotated from $-45^{\circ}$ to $45^{\circ}$ in steps of $1^{\circ}$ to get a rough estimate of rotation angles. The angle of each line is further refined by the same procedure rotating of $\pm 1^{\circ}$ of the rough angle with a step size of $0.025^{\circ}$. All images investigated had a resolution of $640 \times 512$. Thus, in our case, the $\mathbf{R} \in \mathbb{R}^{r d}$ matrix has a size of $r=91 \times d=640$ (for projection on the $x$-axis) or $d=512$ (for projection on the $y$-axis) during the first rough estimation and $r=81 \times d=640$ or $d=512$ during the refinement.

1) Panel Corners: In this scenario, it is assumed that the considered panel is the only object present in the image. Therefore, the lines corresponding to the panel borders are identified as the first and last peaks in the maximization vector for both the $x$ - and 


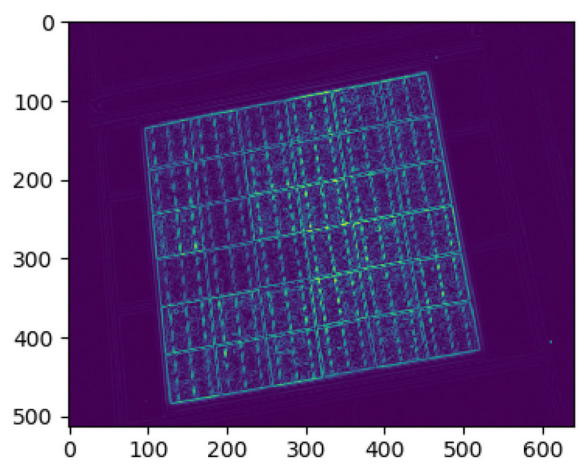

(a)

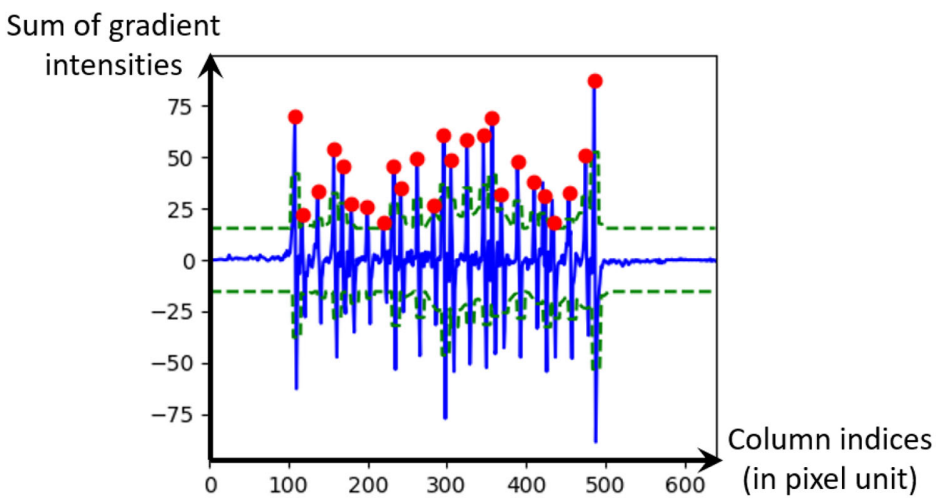

(c)
Rows indices

(in pixel unit)

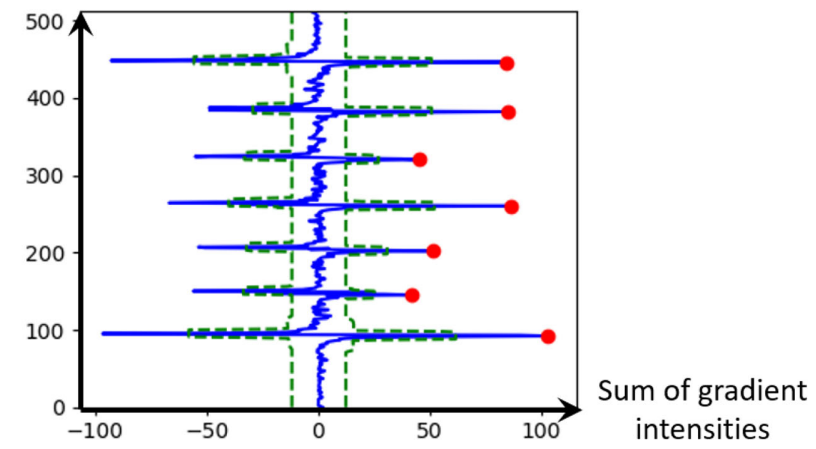

(b)

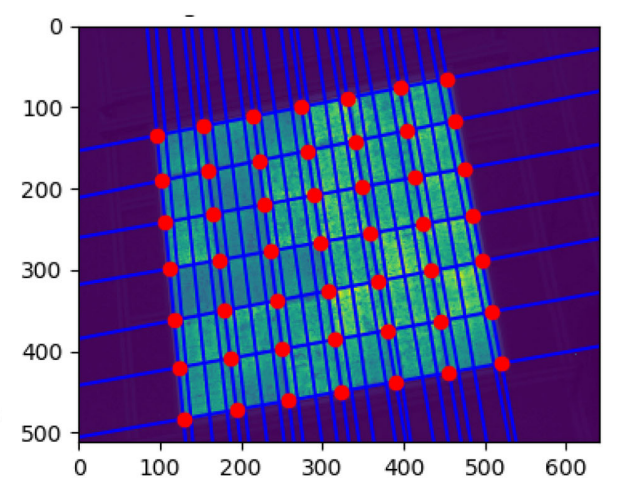

(d)

Fig. 1. Cell corners finding process, as explained in Section III-A. (a) Edge image (axes in pixel units). (b) Maximization vector of (a) on the $y$-axis with found peaks. (c) Maximization vector of (a) on the $x$-axis with found peaks. (d) Resulting lines and cell corners detected.

$y$-axes. A total of four lines are extracted in this manner, and the panel corners correspond to the intersection between them.

2) Cell Corners: The lines corresponding to the cell borders are identified by locating all the peaks within the maximization vector $\boldsymbol{V}_{x \text { or } y}$, instead of a subset (first and last) in the previous case. This results in a greater amount of lines sometimes even including bus bars. The algorithm developed takes as input the number of columns and rows of cells. Sorting cell borders from bus bars is carried out by using all of the vertical lines extracted. Cell borders are then selected among them by assuming that both outermost lines are cell borders and that the distance between cell borders is constant. Fig. 1 illustrates that process.

\section{B. Homography Estimation and Angle Retrieval}

The OpenCV library [16] was used in order to estimate the homography $\mathbf{H}$ [see (1)] from the extracted known points. In this work, we used the findHomography function, which implements the direct linear transformation in combination with the Least Median of Squares method [17] to compute the most probable homography transformation. The identified panel/cell corners along with corresponding points in a rectified image are given as inputs. Points in the rectified image are computed such that all cells have a size of $300 \times 300$ pixels (i.e., a $6 \times 6$ module is approximately $1800 \times 1800$ pixels).

The computed homography $\mathbf{H}$ can be decomposed into a rotation matrix $\mathbf{R}$, a translation vector $t$, and a normal vector $n$ according to the following formula [18]:

$$
\mathbf{H}=\mathbf{R}+t \cdot n^{\top} .
$$

After decomposing the homography according to (2), the rotation matrix $\mathbf{R}$ is used to estimate the camera angles at which the image was captured along the $x-, y$-, and $z$-axes by utilizing Rodrigues' rotation formula [19].

\section{Performance Evaluation}

The evaluation of the two methods presented here is performed in two ways: qualitatively on the perspective correction and quantitatively on the angle estimation. This section first introduces the validation dataset acquired in laboratory settings in Section IV-A. Afterward, the efficiency of the perspective correction is assessed in Section IV-B. Then, the performance of the estimations of the rotation angles is evaluated in Section IV-C. Finally, a "real-life" test is performed on images acquired with a drone in Section IV-D. 
TABLE I

Overview of the Distance and ANGLes Present in the Validation Dataset

\begin{tabular}{|c|c|c|c|c|}
\hline $\begin{array}{c}\text { Distance to } \\
\text { panel (in } \mathrm{cm} \text { ) }\end{array}$ & Rotation $x$-axis & Rotation $y$-axis & Rotation $z$-axis & $\begin{array}{l}\text { Number of } \\
\text { measurements }\end{array}$ \\
\hline 300 and 425 & $0^{\circ}$ & $0^{\circ}$ & $0^{\circ}$ & 2 \\
\hline 300 & $\pm 2^{\circ}, \pm 5^{\circ}, \pm 10^{\circ}, \pm 15^{\circ}, \pm 20^{\circ}, \pm 25^{\circ},+30^{\circ}$ & $0^{\circ}$ & $0^{\circ}$ & 13 \\
\hline 300 & $0^{\circ}$ & $\pm 2^{\circ}, \pm 5^{\circ}, \pm 10^{\circ},+15^{\circ},+20^{\circ},+25^{\circ},+30^{\circ}$ & $0^{\circ}$ & 10 \\
\hline 300 & $0^{\circ}$ & $0^{\circ}$ & $\pm 5^{\circ}, \pm 10^{\circ}, \pm 15^{\circ}$ & 6 \\
\hline 350 & $-2^{\circ},-20^{\circ}$ & $\pm 2^{\circ}, \pm 10^{\circ},+20^{\circ}$ & $0^{\circ}$ & 10 \\
\hline 350 & $\pm 2^{\circ}, \pm 20^{\circ}$ & $0^{\circ}$ & $+5^{\circ},+10^{\circ},+15^{\circ}$ & 12 \\
\hline 350 & $\pm 20^{\circ}$ & $0^{\circ}$ & $\pm 5^{\circ}, \pm 15^{\circ}$ & 4 \\
\hline 350 & $0^{\circ}$ & $2^{\circ}, 20^{\circ}$ & $\pm 5^{\circ}, \pm 10^{\circ}, \pm 15^{\circ}$ & 12 \\
\hline 425 & $\pm 2^{\circ}, \pm 20^{\circ}$ & $2^{\circ}, 20^{\circ}$ & $+5^{\circ},+10^{\circ},+15^{\circ}$ & 24 \\
\hline 425 & $\pm 2^{\circ}, \pm 20^{\circ}$ & $2^{\circ}, 20^{\circ}$ & $-5^{\circ},-15^{\circ}$ & 16 \\
\hline 425 & $\pm 20^{\circ}$ & $2^{\circ}, 20^{\circ}$ & $-10^{\circ}$ & 4 \\
\hline
\end{tabular}

\section{A. Validation Dataset}

The indoor imaging laboratory at Aalborg University was used to acquire the images from the validation dataset. The structure has the capability to image a panel with a $x$-axis rotation in the interval $(-60: 60)^{\circ}$ with a step size of $1^{\circ}$ covering the range of $120^{\circ}$. The same interval is available in the $y$-axis, and both axes are controlled independently. The setup possibilities are further detailed in [20]. For this dataset, no light was let through, and the ambient light noise is considered negligible.

The panel imaged is a 36-cell Solvis SV36-150 PV panel. The images were acquired using a Goldeye CL-033 SWIR InGaAs camera, which has a resolution of $640 \times 512$ and a bit depth of 14 bits [21]. Two parameters of the acquisition are constant: the forward current bias applied to the module is equal to the value of the short-circuit current from the module's datasheet (7.97A) and the exposure time is fixed at $9 \mathrm{~ms}$. The varying parameters are the angle at which the panel is imaged and the camera-topanel distance. The camera-to-panel distance was adapted to the rotation angle to ensure that the panel was fully acquired during the imaging process. Table I contains a description of all the images acquired for this dataset.

\section{B. Results of the Visual Inspection of the Perspective Correction}

Using the estimated homography transformation $[\mathbf{H}$ from (1)], all images from the validation dataset were rectified. The perspective correction was carried out so that each cell spans $300 \times 300$ pixels resulting in an output image of $1800 \times 1800$ pixels. Visual inspection of these images shows satisfying results and no apparent difference between the two methods (panel or cell corners) apart from minor details. Some of the results from these perspective corrections are visualized in Fig. 2. Since all images from the dataset are of the same panel, averaging all the rectified images into one image should result in a good visual quality image presenting no imaging artifacts. Fig. 3 shows the combined perspective corrections by averaging for both methods. It is visible that both are sharp and clear, even though the module method seems slightly better quality than the
TABLE II

Measures of the ERror Between True Angle And Estimated ANGLe FOR EACH AXIS FOR Two CORNER FINDING METHOdS (PANEL CORNERS AND CELl Corners) AND Two Estimation Methods (HOMOgRAPHY ESTIMATION AND REPROJECTION MINIMIZATION)

\begin{tabular}{c|c||c|c|c||c|c|c|}
\cline { 3 - 8 } \multicolumn{2}{c|}{} & \multicolumn{3}{c||}{ Homography estimation } & \multicolumn{2}{c|}{ Reprojection minimization } \\
\cline { 3 - 8 } \multicolumn{2}{c|}{} & $x$-axis & $y$-axis & $z$-axis & $x$-axis & $y$-axis & $z$-axis \\
\hline \hline \multirow{3}{*}{$\begin{array}{c}\text { Panel } \\
\text { Corners }\end{array}$} & Mean & $2.78^{\circ}$ & $2.64^{\circ}$ & $1.28^{\circ}$ & $2.91^{\circ}$ & $2.74^{\circ}$ & $1.29^{\circ}$ \\
\cline { 2 - 8 } & Std & $1.40^{\circ}$ & $1.55^{\circ}$ & $1.71^{\circ}$ & $1.33^{\circ}$ & $1.55^{\circ}$ & $1.73^{\circ}$ \\
\cline { 2 - 8 } & Max & $5.54^{\circ}$ & $7.32^{\circ}$ & $5.91^{\circ}$ & $5.58^{\circ}$ & $7.37^{\circ}$ & $6.07^{\circ}$ \\
\hline \hline \multirow{4}{*}{$\begin{array}{c}\text { Cell } \\
\text { Corners }\end{array}$} & Mean & $3.26^{\circ}$ & $2.05^{\circ}$ & $1.24^{\circ}$ & $3.15^{\circ}$ & $2.40^{\circ}$ & $1.28^{\circ}$ \\
\cline { 2 - 8 } & Std & $2.46^{\circ}$ & $2.06^{\circ}$ & $1.62^{\circ}$ & $1.45^{\circ}$ & $1.87^{\circ}$ & $1.73^{\circ}$ \\
\cline { 2 - 8 } & Max & $9.905^{\circ}$ & $6.23^{\circ}$ & $5.90^{\circ}$ & $5.29^{\circ}$ & $7.28^{\circ}$ & $6.00^{\circ}$ \\
\hline
\end{tabular}

cell method, which is due to the incorrect detection of the cell method between bus bars and vertical cell borders in a few cases.

\section{Results of the Estimation of the Rotation Angles}

The difference between the estimated angle by our methods and the true value of the measure has been computed for each image of the dataset according to (2). Table II contains various measures to quantify this difference over each of the three axes separately: the mean of the absolute difference, the standard deviation, and the maximum.In all cases, the average of the absolute error is significantly smaller along the $z$-axis rotations as compared to $x$ - and $y$-axis rotations. However, the standard deviation is at a similar level for all axes. For the module method, errors on the $x$-axis are negative for $106 / 113$ images (versus $96 / 113$ for the cell method; signs are similar $89 \%$ of the time), while errors on the $y$-axis are positive for 108/113 images (versus $85 / 113$ for the cell method; signs are similar $80 \%$ of the time). Finally, errors on the $z$-axis are positive for $39 / 113$ images for the module method and 38/113 for the cell method, which is estimated nonsignificant. These biases on $x$ - and $y$-axes could be due to under- and overestimation, respectively, of the true angles imaged in the dataset.

Comparison between the panel and cell corner methods yields different appreciation depending on the axis considered. The 

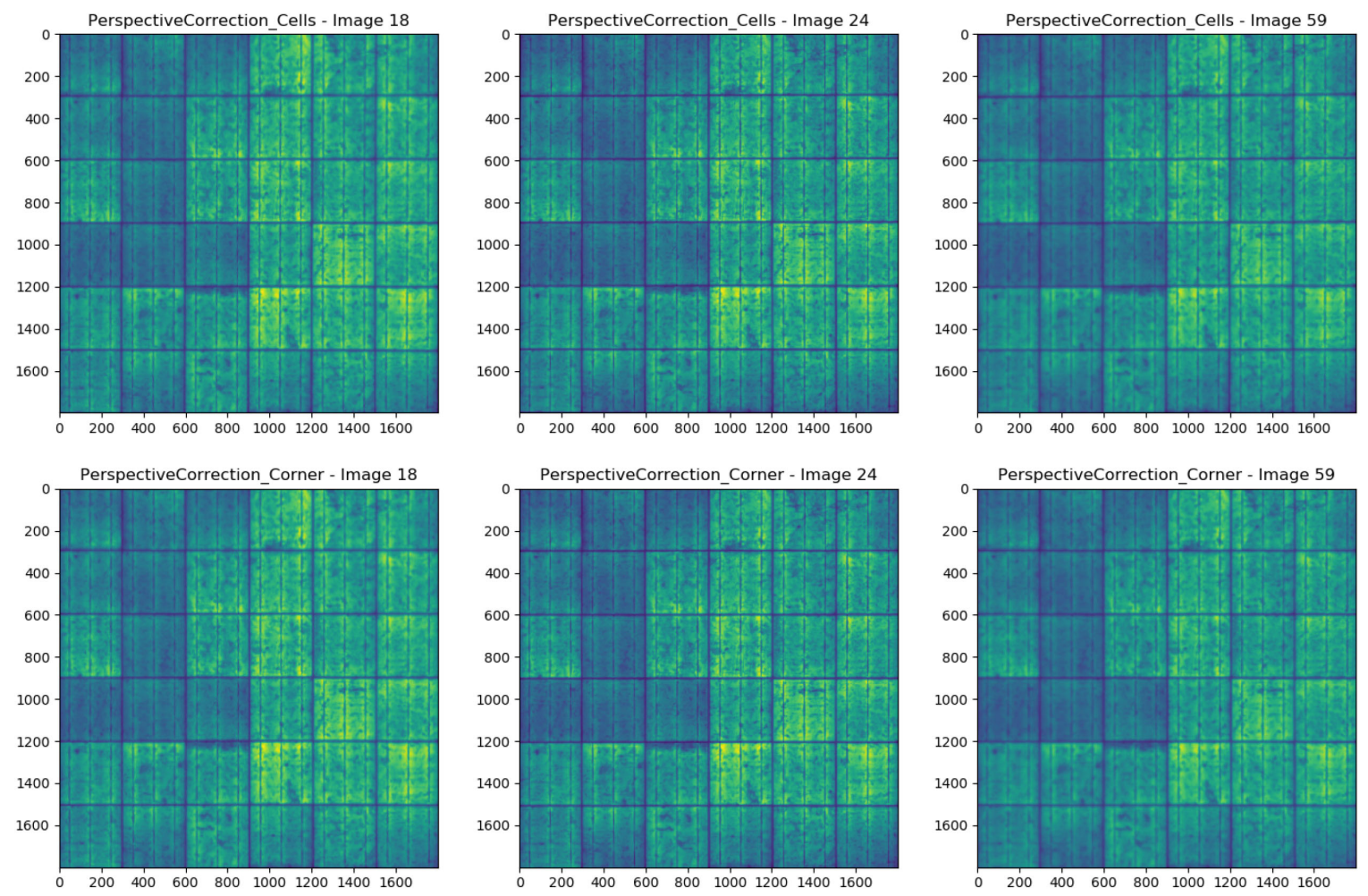

Fig. 2. Resulting perspective corrections of both methods (axes in pixel units).

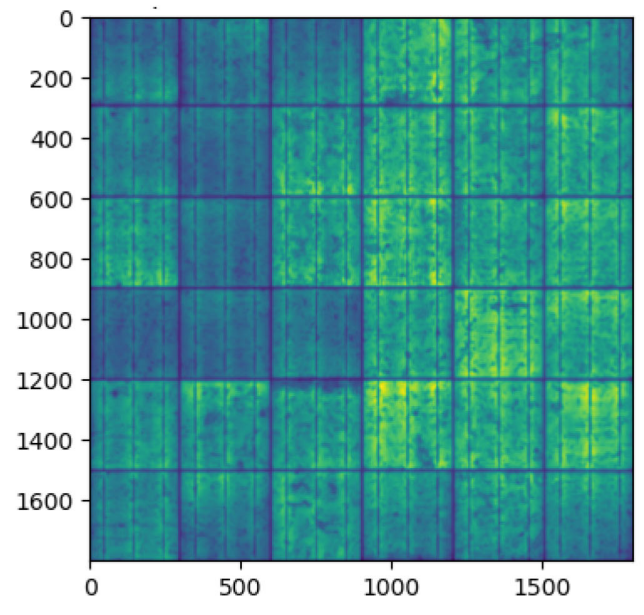

(a)

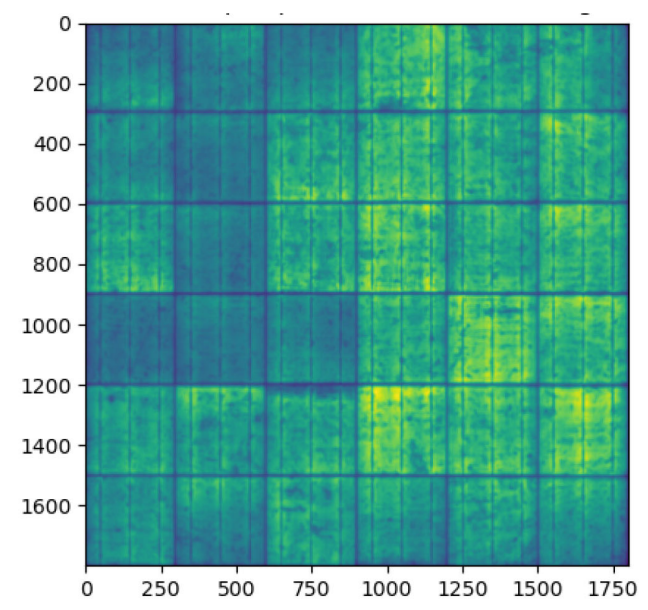

(b)

Fig. 3. Mean of rectified images. (a) Method using panel corners (113 images, axes in pixel units). (b) Method using cell corners (113 images, axes in pixel units).

rotations along the $x$-axis are better estimated by the panel method $\left(2.78^{\circ}\right.$ versus $\left.3.26^{\circ}\right)$. It is the opposite for the $y$-axis ( $2.64^{\circ}$ for the panel method versus $2.05^{\circ}$ for the cell method). Finally, both methods obtain similar average errors along the $z$-axis $\left(1.28^{\circ}\right.$ versus $\left.1.24^{\circ}\right)$.

\section{Test on Real-Life Drone Captured Images}

A test under real conditions was performed on images acquired from a drone embedding a GoldEye CL 33 SWIR camera, as visible in Fig. 4. The test was performed under overcast sky conditions with a maximum irradiance in the horizontal plane of $190 \mathrm{~W} / \mathrm{m}^{2}$. The PV array was forward biased with a square wave current waveform of $20 \mathrm{~Hz}$ and current amplitude equivalent with the short-circuit current of the panels, using a Delta Elektronika SM1500-cp-30 two-quadrant power supply. The camera was equipped with a 25 -mm SWIR lens and a $1150 \mathrm{~nm} \pm 25 \mathrm{~nm}$ OD4 bandpass filter and was programmed to acquire images at $60 \mathrm{frames} / \mathrm{s}$ with and integration time of $2 \mathrm{~ms}$, which allowed obtaining sharp images. All the lines 


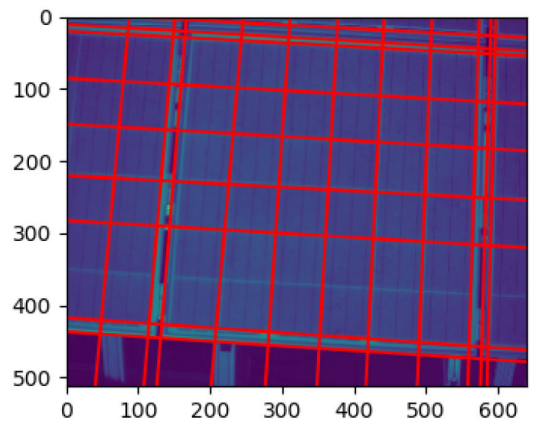

(a)

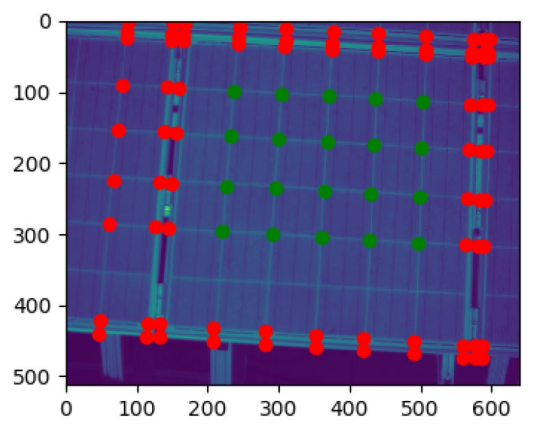

(b)

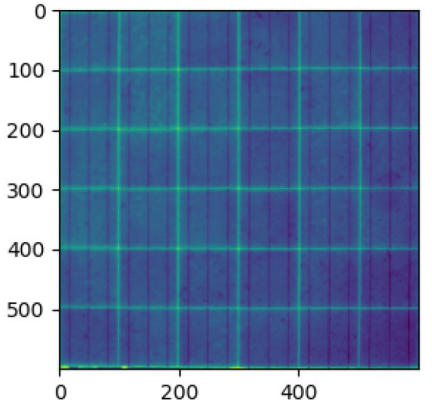

(c)

Fig. 4. Example of perspective correction of an image acquired from a drone. (a) Lines detected (axes in pixel units). (b) Selected points (axes in pixel units). (c) Rectified image (axes in pixel units).

corresponding to the cells' borders are detected except for one, as shown in red in Fig. 4(a) due to the presence of noise from the natural light. To achieve a more robust detection, the method could estimate the spacing between lines and thus refine its selection. As this case corresponds to imaging an array of panels, there are more lines than in the validation dataset, and their pattern is not known a priori. Therefore, among the corresponding intersections, some were manually selected as shown in green in Fig. 4(b). The intersections selected are then used to rectify the image, resulting in Fig. 4(c). For the algorithm to be fully autonomous, an automatic point selection step needs to be added. However, the resulting perspective correction looks satisfying.

\section{CONCLUSION}

In this article, two methods are presented to determine the distortion due to perspective on EL images of PV panels: one using the four panel corners and the other using the corners of each of the cells. A validation dataset composed of 113 images is used for the efficiency evaluation. The performance of those methods is satisfying when applied to rectify the perspective distortion, as visually inspected. The quantitative performance evaluation is variable depending on which rotation axis is considered: the average absolute error is $2.78^{\circ}$ along the $x$-axis, $2.64^{\circ}$ along the $y$-axis, and $1.28^{\circ}$ along the $z$-axis for the panel method and $3.26^{\circ}, 2.05^{\circ}$, and $1.24^{\circ}$ for the cell method. One advantage of the cell method is that it allows further cell-level processing, such as fault detection. As illustrated by a "reallife" condition test, the two methods to detect corners perform well on PV images acquired from a drone, but an additional automatic corner selection is required for a fully autonomous algorithm.

\section{REFERENCES}

[1] A. McCrone, Energy, Vehicles, Sustainability—10 Predictions for 2020, 2020. [Online]. Available: https://about.bnef.com/blog/energy-vehiclessustainability-10-predictio ns-for-2020/

[2] X. Sun, "Solar technology got cheaper and better in the 2010s. Now what?" 2019. [Online]. Available: https://www.greentechmedia.com/articles/ $\mathrm{read} /$ solar-pv-has-become-cheape r-and-better-in-the-2010s-now-what
[3] L. Garcia da Fonseca, "The state of digital O\&M for the solar market," 2019. [Online]. Available: https://www.greentechmedia.com/ articles/read/the-state-of-digital-om-fo r-the-solar-market1

[4] C. Buerhop-Lutz et al., "IR-imaging a tracked PV-plant using an unmanned aerial vehicle," in proc. 32nd Eur. Photovolt. Sol. Energy Conf. Exhib., 2016, pp. 2016-2020.

[5] G. dos Reis Benatto et al., " Drone-based daylight electroluminescence imaging of PV modules," IEEE J. Photovolt., vol. 10, no. 3, pp. 872-877, May 2020.

[6] C. Mantel et al., " SNR study of outdoor electroluminescence images under high sun irradiation," in Proc. 7th World Conf. Photovolt. Energy Convers., 2018, pp. 3285-3289.

[7] C. Mantel et al., "Machine learning prediction of defect types for electroluminescence images of photovoltaic panels," Proc. SPIE, vol. 11139, 2019, Art. no. 1113904.

[8] C. Mantel et al., "Correcting for perspective distortion in electroluminescence images of photovoltaic panels," in Proc. IEEE 7th World Conf. Photovolt. Energy Convers, 2018, pp. 0433-0437.

[9] D. Santana-Cedrés et al., "Automatic correction of perspective and optical distortions," Comput. Vis. Image Understanding, vol. 161, pp. 1-10, 2017.

[10] A. Soycan and M. Soycan, "Perspective correction of building facade images for architectural applications," Eng. Sci. Technol., Int. J., vol. 22, no. 3, pp. 697-705, 2019.

[11] J. Liang, D. DeMenthon, and D. Doermann, "Geometric rectification of camera-captured document images," IEEE Trans. Pattern Anal. Mach. Intell., vol. 30, no. 4, pp. 591-605, Apr. 2008.

[12] K. G. Bedrich, M. Bliss, T. R. Betts, and R. Gottschalg, "Electroluminescence imaging of PV devices: Uncertainty due to optical and perspective distortion," in Proc. 31st Eur. Photovolt. Sol. Energy Conf. Exhib., pp. 1748-1752, 2015.

[13] K. G. Bedrich, M. Bliss, T. R. Betts, and R. Gottschalg, "Electroluminescence imaging of PV devices: Camera calibration and image correction," in Proc. IEEE 43rd Photovolt. Spec. Conf., 2016, pp. 1532-1537.

[14] J. S. Fada et al., "Electroluminescent image processing and cell degradation type classification via computer vision and statistical learning methodologies," in Proc. IEEE 44th Photovolt. Spec. Conf., 2017, pp. 3456-3461.

[15] R. Hartley and A. Zisserman, Multiple View Geometry in Computer Vision. Cambridge, U.K.: Cambridge Univ. Press, 2003.

[16] G. Bradski, "The OpenCV Library," Dr. Dobb's J. Softw. Tools, vol. 25, pp. $120-125,2000$.

[17] P. Rousseeuw, "Least median of squares regression," J. Amer. Statist. Assoc., vol. 79, pp. 871-880, Dec. 1984.

[18] E. Malis and M. Vargas, "Deeper understanding of the homography decomposition for vision-based control," [Research Report] RR-6303, INRIA. 2007, p. 90. [Online]. Available: https://hal.inria.fr/inria-00174036v3

[19] R. Szeliski, Computer Vision: Algorithms and Applications, 1sted. Berlin, Germany: Springer-Verlag, 2010.

[20] H. Parikh et al., "A photovoltaic module diagnostic setup for lock-in electroluminescence imaging," in Proc. 46th IEEE Photovolt. Spec. Conf., Jun. 2019, pp. 538-543.

[21] Goldeye CL-033 TEC1, 2018. [Online]. Available: https://www. alliedvision.com/en/products/cameras/detail/Goldeye/CL-033 20 TEC1. html 